\title{
Systemic Lupus Erythematosus Induced by Isoniazide
}

\author{
Benjelloun Hanane, AMI Meryem", Zaghba Nahid, Yassine Najiba
}

Respiratory Diseases Department, Ibn Rochd University Hospital Center, Casablanca, Morocco

DOI: $10.36347 /$ sjmcr.2020.v08i01.005

| Received: 27.12.2019 | Accepted: 04.01.2020 | Published: 14.01.2020

*Corresponding author: AMI Meryem

\section{Abstract}

Introduction: Systemic lupus erythematosus is an autoimmune disease characterized by the presence of antibodies Nuclear. Drug-induced lupus is an ancient, well-known but rare entity. Observation: We report a case of systemic lupus erythematosus induced by The isoniazid. It was a female Patient, elderly of 23 years old who Introduced lupus induced a month and a half after taking anti-bacillary treatment for pulmonary tuberculosis with positive microscopy. The Disease was manifested by a fever, A Skin impairment made of a facial butterfly rash, scaly erythematic lesions and itchy of arms and legs, without joint, renal, cardiac, or neurological damage. Microcytic hypochromic anemia has been associated with it. Anti-nuclear antibodies and native anti-DNA antibodies were Positive in the serum of the Patient while the anti-Sm, The anti-SSa, The Anti-SSb were absent, anti-histone antibodies were not made for lack of means. Stopping the anti-bacillary treatment Allowed the regression of the lesions after 15 days and their disappearance after two months, as well as the regression of the anti-nuclear antibodies rate after 6 months. The reintroduction of it by discarding the Isoniazide, the most incriminating anti-bacillary, was associated to a good clinical as well as biological tolerance. Thus, the diagnosis of isoniazid-induced lupus was retained. Conclusion: Our case strengthens the possibility of occurrence of drug-induced lupus.

Keywords: Systemic lupus erythematosus; Pulmonary tuberculosis; induced lupus; Isoniazide; supported.

Copyright @ 2020: This is an open-access article distributed under the terms of the Creative Commons Attribution license which permits unrestricted use, distribution, and reproduction in any medium for non-commercial use (NonCommercial, or CC-BY-NC) provided the original author and source are credited.

\section{INTRODUCTION}

Systemic lupus erythematosus is an autoimmune disease characterized by the presence of anti-nuclear antibodies. Induced lupus is defined as a lupus syndrome that can be caused by exposure to a medication or topical agent and can be regressive when stopped. Its frequency continues to increase with the advent of new drugs over the years. Many drugs can induce systemic lupus erythematosus, including Isoniazid, a molecule used throughout the treatment of tuberculosis in Morocco according to the national program fighting against tuberculosis. We report here a case of isoniazid-induced systemic lupus erythematosus.

\section{ObSERVATION}

A 23 year old patient, presented three months before her hospitalization in our hospital service, a bronchial syndrome made of a cough bringing back purulent sputum and several episodes of hemoptysis of low abundance, evolving in a context of febrile sensations, night sweats and deterioration of the general shape. All of the clinical, bacteriological and radiological arguments (fig. 1, a) made it possible to retain the diagnosis of pulmonary tuberculosis with a positive microscopy. Tuberculosis treatment was established according to the national tuberculosis control program, comprising two months of the combination of four combined substances: Isoniazid (H), Rifampicin (R), Ethambutol (E) and Pyrazinamide (P), followed by 4 months of the combination: Rifampicin (R) and Isoniazid (H). A month and a half after the start of treatment, skin lesions appeared in the photo exposed areas such as butterfly wing erythema of the face, scaly and itchy erythema rash of the arms and legs, associated with a fever, without arthralgia or other associated signs. Biological examinations showed an inflammatory syndrome with a sedimentation rate raised to $112 \mathrm{~mm}$, a protein $\mathrm{C}$ reaction (CRP) moderately elevated to $78 \mathrm{mg} / 1$, an hypochromic microcytic anemia (hemoglobin at $8 \mathrm{~g} / \mathrm{dl}$ ), without leukopenia, neither lymphopenia, nor neutropenia. The renal balance was correct. The serum assay of antinuclear antibodies as well as that of native anti-DNA antibodies were positive at 1/320 and 1/80 respectively. The anti-Smith (Sm), antiSSa / Ro, anti-SSb / La, and rheumatoid factor antibodies were negative. The antihistone antibody assay was not carried out for lack of resources. Human immunodeficiency virus, syphilitic, 
hepatitis Band $\mathrm{C}$ serologies were negative. Bacillus Koch's search for sputum was always positive on direct examination. At this stage, the diagnosis of druginduced lupus, including antibiotics, was strongly suspected. We then stopped anti bacillary treatment until the skin signs regressed after one month and then gradually reintroduced the treatment by discarding Isoniazid and starting with the least incriminated antibiotic, including Ethambutol, then Rifampicin and Pyrazinamide. The evolution was favorable with good tolerance to treatment, complete regression of clinical and radiological signs (fig. 1, b) after three months as well as the level of anti-nuclear antibodies during the six following months. This is how the diagnosis of lupus induced by Isoniazid was taken.

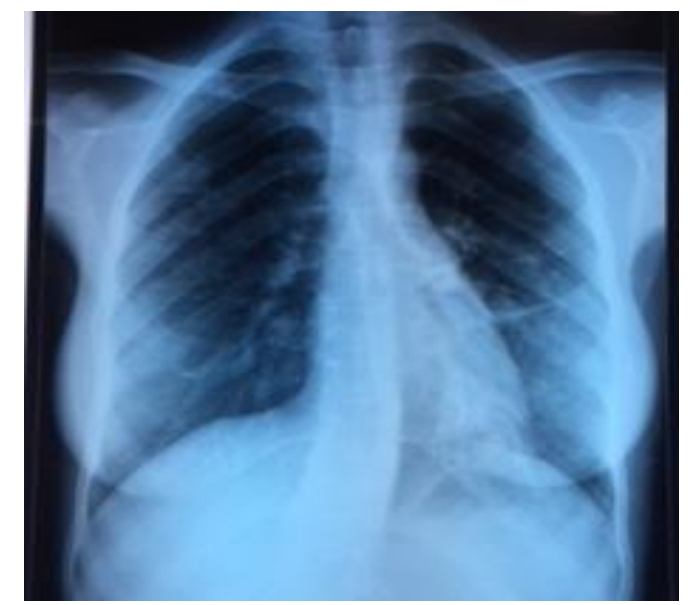

Fig-1: Front chest x-ray (a) excavated opacity with left Hilar projection (before treatment)

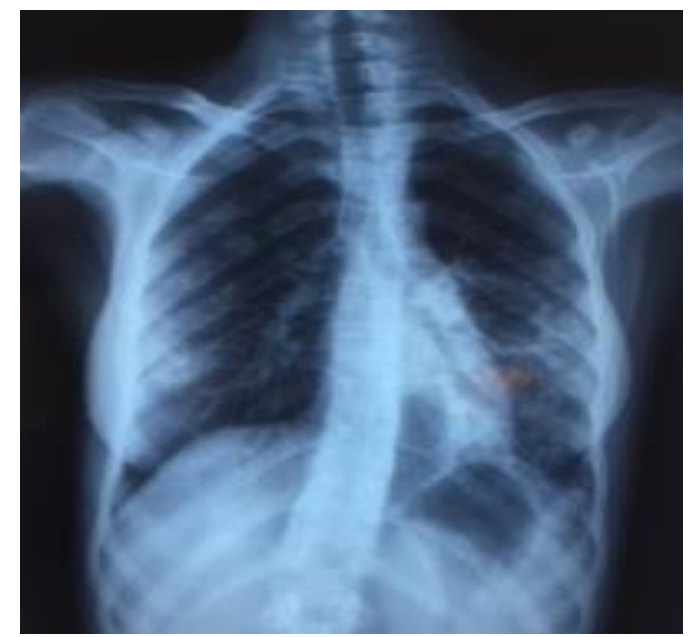

Fig-2: Front chest x-ray (b) persistence of linear interhilo-axillary opacity (after treatment)

\section{DISCUSSION}

Induced lupus is defined as a lupus generally cutaneous-articular syndrome secondary to continuous exposure to a drug and which disappears after being stopped to this exposure. Drug-induced lupus accounts for $10 \%$ of lupus [1]. The first recorded cases were induced by sulfadiazine and hydralazine, reported mainly in 1945 and 1953. More than 80 drugs can induce lupus erythematosus [2]. The most frequently responsible are hydralazine and procainamide. Calcium channel blockers, converting enzyme inhibitors, interferons, thiazide diuretics, terbinafine may also be responsible for subacute skin damage [3]. Among the anti-tuberculosis drugs, isoniazid is most often accused, followed by rifampicin, the two major antibacillaries used throughout the duration of treatment. Although isoniazid is the most common anti-tuberculosis drug, one case of streptomycin-induced lupus has been reported in a 48-year-old woman treated for pulmonary tuberculosis [4].

The literature review has shown that induced lupus occurs in young patients with a female predominance in all cases $[1,5,6]$. Our patient was put on antibacillary treatment for pulmonary tuberculosis with positive microscopy, while in other studies, it was pleural tuberculosis [1,5] and tuberculous meningitis complicating surgical dissemination from 'a potty sickness [6]. In our study as well as that of A. Nezzal et $a l$. The time between the start of antibacillary treatment and the appearance of clinical signs of lupus was two months [6]. It was shorter than a month in JL's study. Rakotoson et al (one month) [1], and longer than 6 months in the study by J. Ben Amar et al. (six months) [5].

Drug-induced lupus erythematosus can be classified into systemic, subacute skin damage and chronic skin damage [2]. The diagnosis is based on four criteria [7]:

- Absence of clinical and biological signs before the administration of the product

- $\quad$ Taking a potentially inducing drug

- Reversibility of symptoms upon cessation of treatment, with variable delays ranging from a few weeks to two years

- Presence of antinuclear antibodies with at least one clinical symptom of systemic lupus.

Isoniazid is a major anti-tuberculosis drug used for over 60 years. The overall frequency of its side effects is estimated at $5 \%$. The latter are mainly represented by liver and neurological damage. Isoniazid-induced lupus occurs in $1 \%$ of cases [8]. It can affect both sexes. Twenty-two percent of patients treated with Isoniazid develop anti-nuclear antibodies on average after six months. According to the authors, the most common symptoms are arthralgia, myalgia, pleurisy, rash and fever associated with anti-nuclear antibodies. Visceral involvement, in particular renal and neurological, during drug-induced lupus erythematosus is rare [3]. It is the severity of the disease and therefore justifies specific management [9]. In our study and in that made in Tunisia [5], the clinical signs revealing the induced lupus were dominated by a specifically lupus cutaneous attack, whereas they were more serious in the study made in Madagascar with neurological and renal 
damage [1]. The biological manifestations are identical to those of systemic lupus erythematosus [5]. The rise in anti-histone antibodies is specific, according to Vázquez-Del Mercado, who conducted a study to determine the prevalence and specificity of these antibodies in tuberculosis patients treated with Isoniazid. This author also concluded that this drug is classified as an inducer of autoimmunity. The presence of anti-histone antibodies in the patient's serum is the consequence of isoniazid stimulation of lymphocytes in the intestinal Peyer's patches or in the intestinal lymphoid follicles [9]. In our study, as in others, the assay of native anti nuclear and anti DNA antibodies was positive $[1,5]$.

The diagnosis of induced lupus involves stopping the inducing treatment, a fortiori, Isoniazide in our observation. The regression of clinical symptoms is generally observed in a few days to a few weeks as in the case of our patient. Biological abnormalities can persist longé (several months). Isoniazid has been discontinued in all studies, thus inducing complete regression of clinical and biological signs within a variable period between one and two months with the negotiation of the immunological assessment after six months $[1,5,6]$. Only the persistence of lupus clinical signs justifies a specific treatment (synthetic anti malarial, corticosteroid therapy, immunosuppressant). It is also necessary to avoid, if possible, the reintroduction of a treatment of the same therapeutic class.

\section{CONCLUSION}

Induced lupus is a rare and little-known complication. Isoniazid is one of the major antituberculosis drugs and represents the most implicated anti-tuberculosis agent in this disease.

\section{REFERENCES}

1. Rakotoson JL, Randriamanana D, Rakotomizao JR, Andrianasolo R, Rakotoarivelo R, Andrianarisoa AC. Severe systemic lupus erythematosus induced by isoniazide. Revue de Pneumologie clinique. 2009;65, 361-364.

2. Sarzi-Puttini P, Atzeni F, Capsoni F, Lubrano E, Doria A. Drug- induced lupus erythematosus. Autoimmunity. 2005;38:507-18.

3. Vedove CD, Del Giglio M, Schena D, Girolomoni G. Drug-induced lupus erythematosus. Arch Dermatol Res. 2009;301:99-105.

4. Toyoshima M, Taniquchi M, Imokawa S, Nakazawa K, Sato A, Hayakawa H. A case of streptomycin-induced lupus. 1993; 68:319-23.

5. Ben Amar J, Dhahri B, Smadhi H, Azzabi S, Baccar M A, Aouina H. Lupus érythémateux systémique induit par l'isoniazide, Revue française d'allergologie. 52; 2012: 462-464 .

6. Nezzal A, Louanchi M, Lakehel M, Toubal N. Lupus induit par l'isoniazide : à propos d'un cas. revue neurologique. 2013, A164-A175.

7. Hachulla E, Flipo RM, Puisieux F. Rhumatismes inflammatoires induits par les médicaments. Rev Rhum (Ed Fr) 2004;71:489-97.

8. Yee D, Valiquette C, Pelletier M, Rocher I, Menzies D. Incidence of serious side effects form first-line antituberculosis drugs among patients treated for active tuberculosis. Is J Respir Crit Care Med. 2003; 167:1472-7?

9. Vázquez-Del Mercado M, Casiano CA, Rubin RL. $\operatorname{IgA}$ antihistone antibodies in isoniazid-treated tuberculosis patients. Autoim- munity. 1995;20:105-11. 